Argumentation Argumentation et Analyse du Discours

\title{
Faire entendre les sans-voix
}

Bringing the voice of the voiceless

\section{Dominique Maingueneau}

\section{(2) OpenEdition}

\section{Journals}

\section{Electronic version}

URL: http://journals.openedition.org/aad/4131

DOI: 10.4000/aad.4131

ISSN: 1565-8961

\section{Publisher}

Université de Tel-Aviv

\section{Electronic reference}

Dominique Maingueneau, «Faire entendre les sans-voix », Argumentation et Analyse du Discours [Online], 24 | 2020, Online since 16 April 2020, connection on 20 April 2020. URL : http:// journals.openedition.org/aad/4131; DOI : https://doi.org/10.4000/aad.4131

This text was automatically generated on 20 April 2020.

\section{(c) $($ ) $(9)$}

Argumentation \& analyse du discours est mis à disposition selon les termes de la licence Creative Commons Attribution - Pas d'Utilisation Commerciale - Pas de Modification 4.0 International. 


\title{
Faire entendre les sans-voix
}

\author{
Bringing the voice of the voiceless
}

\section{Dominique Maingueneau}

\section{Introduction}

1 Le thème de cet article se trouve au croisement de l'analyse du discours, de la rhétorique et d'une problématique récente dans les sciences sociales, celle de la « vulnérabilité » (Brodiez-Dolino et al. éds, 2014; Thomas 2010; Maillard 2011), qui entretient des relations étroites avec d'autres, comme la réflexion sur les «acteurs faibles» (Payet, Giuliani, Laforgue éds 2008; Payet 2011), le "care» (Tronto 2009 [1993]), certains courants des gender studies (Butler 2004 [1997]) ou, plus anciennement, la réflexion sur le «subalterne » de D. Spivak (2006 [1988]). L'analyse du discours, de son côté, s'est intéressée à la parole des "sans - (Guilhaumou 1998) et a engagé une réflexion d'ordre éthique et politique à propos de la relation que les analystes entretiennent avec eux (Rabatel 2016; Paveau 2017); un colloque a été organisé récemment à l'Université Paris-Est Créteil sur le thème "Donner la parole aux "sansvoix" ? Acteurs, dispositifs et discours $»^{1}$. Notre contribution s'inscrit, on le voit, dans un domaine de recherche particulièrement actif, symptomatique d'une époque où l'on ne cesse d'opposer des dominants à des sujets radicalement problématiques qu'on qualifie, selon le point de vue que l'on adopte, de "faibles", de "sans - ", de "démunis », d'« exclus», de "pauvres», de "périphériques", d'« invisibles», de " minorés », de « subalternes »...

2 A la différence des approches de type sociologique, nous allons fixer notre attention sur une question d'ordre discursif, en l'occurrence les difficultés que soulève la traduction de leurs paroles par des médiateurs soucieux d'attirer sur eux la pitié 2 de ceux qui sont étrangers à leur monde. Au niveau où nous nous situons ici, il n'est pas nécessaire de faire une distinction entre "pitié » et «compassion». Notre propos ne porte pas sur l'émotion elle-même mais sur la médiation entre des personnes présentées comme dignes de pitié et un public qu'il faut mobiliser en leur faveur. 
Quand on traite ainsi d'appel à la pitié, on peut distinguer entre l'appel à la pitié comme argument parmi d'autres au sein d'un texte, par exemple une plaidoirie, et l'appel à la pitié comme visée qui anime l'ensemble du texte. C'est ce dernier cas qui nous intéresse ici. Si la pitié et les appels à la pitié traversent les âges, les dispositifs d'énonciation par lesquels ils passent, eux, varient. La notion d'espace public elle-même n'a rien d'intemporel : sa configuration actuelle est bien différente de celle qui prévalait il y a quelques dizaines d'années. Aujourd'hui un texte d'appel à la pitié met en relation un locuteur et un public qui consulte divers médias connectés entre eux (presse écrite, sites web d'information, télévision, radio, réseaux sociaux) pour défendre une personne ou une collectivité - des souffrants - qui, présentés comme des "sans-voix», ont crucialement besoin d'un médiateur.

Plutôt que d'étudier des genres conçus spécifiquement pour appeler à la pitié, comme les affiches ou les clips produits par des organisations (ONG caritatives, agences, ministères...) en charge de certains dysfonctionnements sociaux, nous allons nous intéresser aux frontières du registre de l'appel à la pitié. Nous soulignerons ainsi la différence avec les médiations de type politique, avant de nous concentrer plus longuement sur les difficultés auxquelles se heurte l'appel à la pitié quand il mobilise des ressources d'ordre esthétique.

On ne peut aborder cette question en considérant le seul texte, sans prendre en compte l'ensemble du dispositif d'énonciation, en particulier le genre de discours et le statut du locuteur, sa relation $\mathrm{au}(\mathrm{x})$ souffrant(s). Un poème de Victor Hugo particulièrement émouvant, "Souvenir de la nuit du 4 » (Châtiments II, 3), évoque la veillée funèbre d'un enfant, tué lors du coup d'Etat de Louis-Napoléon Bonaparte. C'est un appel à la pitié manifeste pour les victimes de ce coup d'Etat ; mais c'est aussi un texte de dénonciation politique et un poème dans un recueil soigneusement composé. Ici littérature, politique et appel à la pitié sont inextricablement noués. Mais il est facile de voir que les trois visées ne sont pas sur le même plan, que l'appel à la pitié est subordonné. Notre propos consiste au contraire à caractériser des énonciations où l'appel à la pitié se trouve au premier plan, en nous limitant aux situations où s'institue un porte-parole de personnes dont la parole n'est pas audible

6 Nous allons ainsi mettre en évidence ce que l'on peut appeler la « zone de variation » de l'appel à la pitié, quand ce dernier ne bascule pas dans un autre registre. La comparaison avec le politique s'impose, dans la mesure où c'est un type de discours qui se donne pour tâche d'assurer le bien-être de l'ensemble de la population. Prendre acte de sa spécificité, c'est tracer une limite au-delà de laquelle l'appel à la pitié change de nature. Quant à l'esthétique, elle définit une autre limite : celle par laquelle l'appel à la pitié se muerait en œuvre, avec toutes les conséquences d'ordre pragmatique que cela entraîne. Mais cette frontière est difficile à tracer car les ressources esthétiques jouent un rôle essentiel quand il s'agit de toucher la sensibilité.

7 Nous ne procéderons pas en nous appuyant sur un corpus mais nous illustrerons quelques distinctions de base à l'aide d'exemples qui nous paraissent emblématiques. De toute façon, on voit mal comment constituer un corpus à ce sujet sans présupposer ces distinctions. 


\section{Sphère haute, sphère basse}

8 Les représentations communes en matière de circulation des énoncés impliquent une différence entre une sphère qu'on pourrait dire « haute » et une sphère «basse ». Un grand nombre de gens expriment en effet le sentiment de " ne pas être entendus " par ceux qui ont le pouvoir d'agir. Leur parole leur apparaît doublement basse : venue d'en bas, de gens sans importance, elle n'est pas entendue, comme s'ils parlaient «à voix basse ». S'impose alors la nécessité du médiateur qui pourra les rendre audibles, faire accéder leur point de vue à la sphère haute. Il va de soi que cette distinction entre deux sphères ne repose sur aucun critère sociologique sérieux, mais elle joue un rôle important dans l'imaginaire collectif et sert d'argument dans les énonciations de type revendicatif.

9 En revanche, dès que l'on a affaire à des médiateurs qui se réclament de l'espace politique au sens large, «ne pas être entendu » prend un sens différent. On le voit dans cet extrait du site de la C.F.D.T., l'un des deux grands syndicats français :

La nouvelle loi Travail fait donc débat et une journée de mobilisation se prépare pour mercredi. Reçu par Manuel Valls dans l'après-midi, Laurent BERGER est bien décidé à faire évoluer ce texte et il l'assure : « il y aura une mobilisation si nous ne sommes pas entendus ${ }^{3} »$. Il a, en effet expliqué faire "partie des $70 \%$ de Français qui sont opposés à l'écriture de la loi telle qu'elle est aujourd'hui. Il faut la retravailler, c'est mon état d'esprit et c'est ce que je vais dire au Premier ministre aujourd'hui" (texte mis en ligne le 9 mars 2016, (http://www.cfdt-interco21.fr/actualites/540-projet-de-loi-travail-il-y-auraune-mobilisation-si-nous-ne-sommes-pas-entendus.html ; consulté le 9-10-2018)

10 Avec un autre premier ministre et d'autres opposants, en l'occurrence les «Gilets jaunes", la valeur du verbe «entendre " reste la même. L'agent est toujours le gouvernement et le patient les locuteurs qui contestent sa politique :

Les annonces faites ce mardi par le Premier ministre n'ont pas rencontré un écho favorable du côté du rond-point de Gohélève où une vingtaine de Gilets jaunes ont maintenu leurs barrages filtrant. « Il ne propose qu'un moratoire. Et après? Rien ne changera ", affirme l'un des manifestants. " Il n'y a aucune proposition concernant le pouvoir d'achat. Il ne nous a pas entendus », ont lancé des retraités, déçus mais déterminés (Le Télégramme, 4/12/2018, (https://www.letelegramme.fr/morbihan/pontivy/gilets-jaunes-le-premierministre-ne-nous-a-pas-convaincus-04-12-2018-12152376.php Consulté le 27/12/2018)

11 Il s'agit là d'une formule rituelle en France dans des contextes de lutte syndicale ou politique: «n'être pas entendu », c'est être perçu (la CFDT est reçue par le premier ministre, les «Gilets jaunes » ont contraint le premier ministre à leur répondre), mais considérer que sa parole, contre toute justice, n'a pas été réellement prise en compte, suivie d'effets. Le recours à la construction hypothétique, "si nous ne sommes pas entendus... ", prend alors valeur de menace à l'égard de l'adversaire. C'est toute la différence entre le conflit politique, qui suppose une relation entre des actants situés dans un même espace, et l'appel à la pitié où les souffrants qui «ne sont pas entendus » sont censés se trouver à l'extérieur du monde partagé par le locuteur et son public. 
Il arrive que cette divergence entre la revendication et la pitié soit soulignée par les acteurs politiques eux-mêmes, par exemple quand ils opposent la « charité » à la prise en compte de leurs "droits ", nécessairement " légitimes ». C'est le cas dans cet extrait d'un débat sur la politique pour les territoires d'Outre-mer à l'Assemblée nationale ; un député du parti d'extrême-gauche la «France insoumise », J.-H. Ratenon, s'exprime ainsi :

L'État sait cependant que les outre-mer possèdent de multiples atouts pour se développer, comme cela a été encore rappelé aujourd'hui : l'agriculture, la biodiversité, le tourisme, la culture, l'économie bleue, les énergies renouvelables, la recherche, et j'en passe... mais surtout une population de plus en plus qualifiée et motivée. On a rappelé que la France possède le deuxième domaine maritime mondial : c'est une richesse qui lui est offerte par l'outre-mer! Nous ne réclamons pas la charitée, mais l'État a le devoir de nous donner les moyens de réussir notre développement. Quand et comment allez-vous faire suffisamment confiance aux outre-mer pour déclencher véritablement leur développement et reconnaître concrètement leur spécificité?

(Séance du mercredi 21 février 2018, « Questions sur la politique économique en outre-mer", http://www.assemblee-nationale.fr/15/cri/ 2017-2018/20180148.asp\#P1193286 consulté le 3/1/2019).

13 La première partie de l'argumentation s'appuie sur un verbe factif («l'Etat sait que...»), c'est-à-dire un verbe qui présuppose la vérité des complétives qui le suivent. L'orateur recense les "atouts" dont dispose l'Outre-mer pour instaurer une sorte de contrat entre deux partenaires en s'appuyant sur le topos de l'égalité de traitement entre tous les citoyens d'un même pays. Il entend ainsi justifier l'énonciation de "Nous ne réclamons pas la charité ", énoncé étayé par la norme selon laquelle «l'État a le devoir de nous aider... ".

Le médiateur d'un appel à la pitié pour des "sans-voix ", lui, ne s'autorise pas d'un contrat de nature politique, mais d'une commune appartenance à la condition humaine et de sa relation personnelle avec les souffrants qu'il défend. Ce contact peut prendre des tours variés. Il peut même relever d'une routine professionnelle. C'est le cas lorsque des journalistes tendent un micro à des souffrants chroniques (vieillards sans famille, habitants de bidonvilles, travailleurs exploités...), ou ponctuels (catastrophes naturelles, incendies, guerres, etc.). En règle générale, les journalistes s'efforcent de faire oublier leur présence et de ne pas afficher une relation personnelle avec ces souffrants : il s'agit avant tout de leur donner la parole. L'appel à la pitié tend alors à être indirect: le seul fait de s'intéresser à ces personnes, le choix des locuteurs pertinents, le cadrage verbal et/ou visuel, le montage de la séquence doivent suffire à susciter la pitié. Il n'est pas rare que les médias indiquent en conclusion les coordonnées d'une organisation caritative à laquelle il est possible de faire des dons. Il y a ici complémentarité entre la tâche du journaliste et celle des humanitaires.

15 Ce n'est pas ce type de mise en scène du pathos, abondamment étudié par les spécialistes des médias ${ }^{5}$, qui nous intéresse ici, mais les situations où il y a engagement fort en faveur des souffrants, où quelqu'un montre à la face du monde qu'il parle pour un individu ou un groupe qui n'auraient pas accès à la sphère haute. La préposition " pour » a ici la double valeur de " à la place de » et « en faveur de ». qui ne se laisse pas enfermer dans l'univers politique: n'importe quelle entité déjà 
constituée - individu ou collectivité - peut désigner quelqu'un pour parler en son nom ${ }^{6}$. Ce qui pose un problème juridique : à quelles conditions, $\mathrm{X}$ est-il en droit de parler au nom de $Y$ ?, mais aussi, pour l'analyste du discours, une question radicale : « comment présenter dans la parole dite ceux qui, par principe, ne peuvent y être présents?» (Gautier $2015: 3$ ) Ce modèle classique du porte-parole peut difficilement s'appliquer quand il y a appel à la pitié en faveur de sans-voix : ceux-ci ne constituent pas à proprement parler une collectivité organisée, et ils n'établissent pas de relation contractuelle avec un mandataire. Il apparaît ainsi utile d'établir une distinction à l'intérieur de la catégorie des porte-paroles entre les délégués et les inspirés, étant entendu qu'il arrive que les deux se mêlent dans des proportions variables. Le porteparole «inspiré » prétend s'appuyer non sur un mandat, une organisation et des procédures de nomination, mais sur des motivations d'ordre éthique - peu importe quelle en est la nature -, et souvent même sans que ceux en faveur de qui il parle le lui aient demandé. Quand il entend parler pour les « sans-voix », il peut être dit porte-voix. Ce nom composé, sans-voix, s'inscrit dans un paradigme lexical particulièrement productif aujourd'hui : les " sans domicile fixe », « sans-papiers », « sans-logis », « sansabri », « sans-logement », « sans-droits », « sans-travail », « sans-terre », « sans-Etat » ${ }^{7}$ ... Le lexème "sans-voix " permet d'ailleurs de subsumer l'ensemble de ces "sans - ", dès lors que ces derniers sont perçus comme exclus de la sphère haute de la parole.

Si le sans-voix n'est pas audible, il se voit néanmoins attribuer la Voix par excellence, par sa souffrance même. A la différence de la parole, la voix est au plus près de la nature : parfois pur cri, en-deçà de toute articulation, pure expression d'une intériorité. Elle n'est pas altérée par les multiples contraintes auxquelles est soumise l'énonciation quand elle s'exerce dans la sphère haute.

\section{2. «Le porte-parole des sans-voix »}

19 Dès lors qu'il lui faut énoncer dans la sphère haute au nom de personnes qui en sont censées exclues, la position du porte-voix s'avère particulièrement délicate. Il lui faut d'une manière ou d'une autre montrer par ses gestes mêmes, et au premier chef par son énonciation, qu'il n'interpose pas sa personne entre les souffrants et le public, que sa parole ne se replie pas sur elle-même mais se trouve en prise sur celle des sans-voix qu'elle doit porter. Le problème, on l'a dit, est qu'il lui faut aussi se soumettre aux contraintes des genres de discours spécifiques de la sphère haute. Il doit rendre audible et recevable la voix qu'il porte, mais sans altérer son innocence originaire.

Même les énonciations politiques, qui par nature récusent l'appel à la pitié, sont prises dans cette difficulté, quand les militants se présentent comme parlant au nom d'exclus de la sphère haute. Par nature, le discours politique se développe dans un champ soumis à des contraintes spécifiques qui risquent d'altérer la relation aux souffrants. Le soupçon pèse constamment sur les politiques d'exploiter certaines souffrances «à des fins politiciennes" ou de "ne pas être suffisamment proches» des populations défavorisées. C'est à travers la façon dont ils s'inscrivent dans le discours que les hommes ou les femmes politiques peuvent espérer répondre à ces critiques récurrentes.

21 Considérons par exemple ce texte de presse - extrait du site d'un journal en ligne de l'île de la Réunion - qui présente les candidats du Parti Communiste à des élections 
locales. La lexie "porte-parole des sans-voix », détachée dans le titre, reprend une expression des candidats eux-mêmes.

Les porte-parole des « sans voix »

David Lebon et Betty Grondin, candidats PCR, ont présenté hier, aux côtés de leurs remplaçants Joël Vienne et Catherine Lejoyeux, leur programme aux élections départementales sur le canton 13, commune de Saint-Joseph. Ils souhaitent se positionner comme «les porte-parole des sans voix. Il est fini le temps où ce sont les technocrates qui décident, les autres et les jeunes doivent agir. Nous voulons fédérer et rassembler toutes les bonnes volontés pour travailler et défendre les intérêts de ce canton, avec disponibilité, proximité, rigueur, et efficacité. Cette élection, offre à la population l'occasion de s'exprimer ", a déclaré d'emblée David Lebon.

Le programme du binôme s'articule sur un triptyque: jeunesse/avenir, proximité, et solidarité. L'accent est mis également sur le volet social. "Encore aujourd'hui au XXIe siècle, à Saint-Joseph, des personnes vivent dans la précarité et l'insalubrité, sans eau ni électricité. Je veux œuvrer à leur côté en utilisant tous les moyens du conseil départemental qui, je vous le rappelle, à pour compétence l'action sociale. Je me pose la question de savoir quel est le bilan du conseiller général sortant, qui est également adjoint au maire, et administrateur de la Sodegis. Le comble est tout de même que, jusqu"à maintenant, beaucoup de citoyens ne savent toujours pas qui est leur conseiller général ", a tenu à souligner Betty Grondin.

(http://www.clicanoo.re/?page=archive.consulter\&id_article=461915 ; consulté le 8/2/2019; l'italique est de nous).

Le texte construit une opposition entre les "sans voix", placés en bas, et "les "technocrates qui décident ", en haut. Terme évaluatif négatif, technocrate désigne, rappelons-le, un "personnage politique ou haut fonctionnaire qui agit, décide en fonction de données techniques ou économiques et sans donner la priorité aux facteurs humains " (Trésor de la Langue Française, article «technocrate »). Ceux qui s'instituent en "porte-parole des sans voix " s'attachent en général à montrer à divers indices qu'ils ont un contact privilégié avec eux, voire, quand c'est possible, qu'ils sont des leurs, au moins pour une part de leur existence. C'est ce qui ressort par exemple de la photo associée au texte.

POLITIQUE

\section{Les porte-parole des " sans voix »}

ॠ Réagir | Clicanoo.com | publié le 17 février 2015 | 02h30

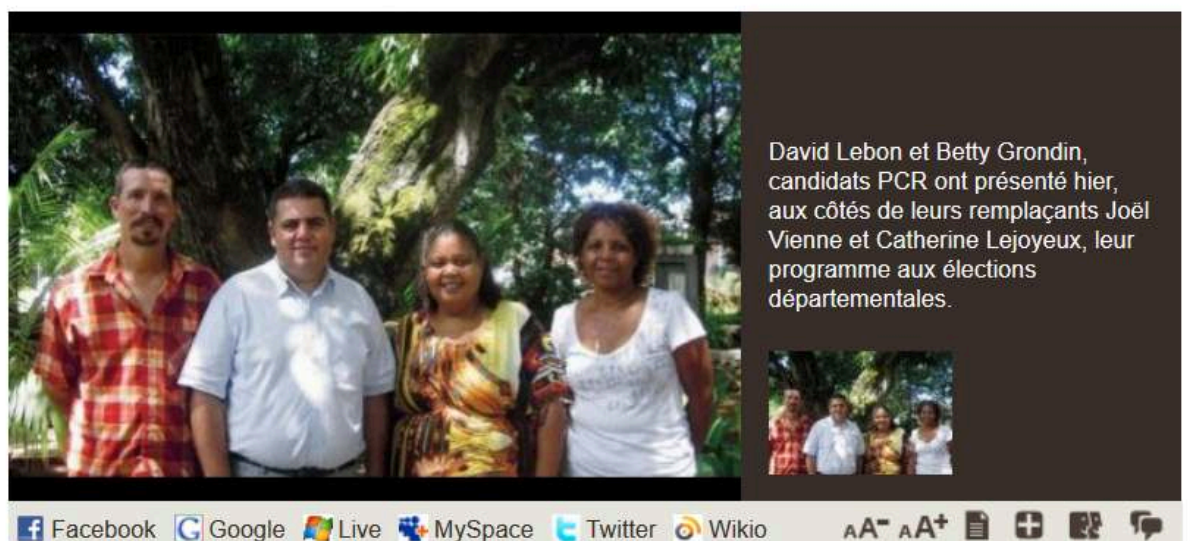



naturel, tout cela contribue à activer chez le lecteur un ethos de simplicité, témoignage d'une proximité avec une vérité, une nature. C'est particulièrement pertinent pour une élection locale, qui engage des enjeux liés à la vie quotidienne des citoyens. La légitimité des candidats en dépend pour une part importante. Le médiateur entend montrer qu'il est au contact des sans-voix, tout en étant membre d'une organisation politique qui a accès à la sphère haute.

Cette contrainte ne pèse pas de manière égale sur tous les partis, mais avant tout sur ceux qui se posent en porte-paroles des exclus. On peut comparer, de ce point de vue, les images des candidats que proposaient aux élections européennes de 2009 les professions de foi ${ }^{8} \mathrm{du}$ MODEM, parti centriste qui tient un discours qui s'adresse à l'ensemble des électeurs, et le Nouveau Parti Anticapitaliste (NPA), qui se pose en porte-parole des victimes du capitalisme. Voici la première page de son texte :

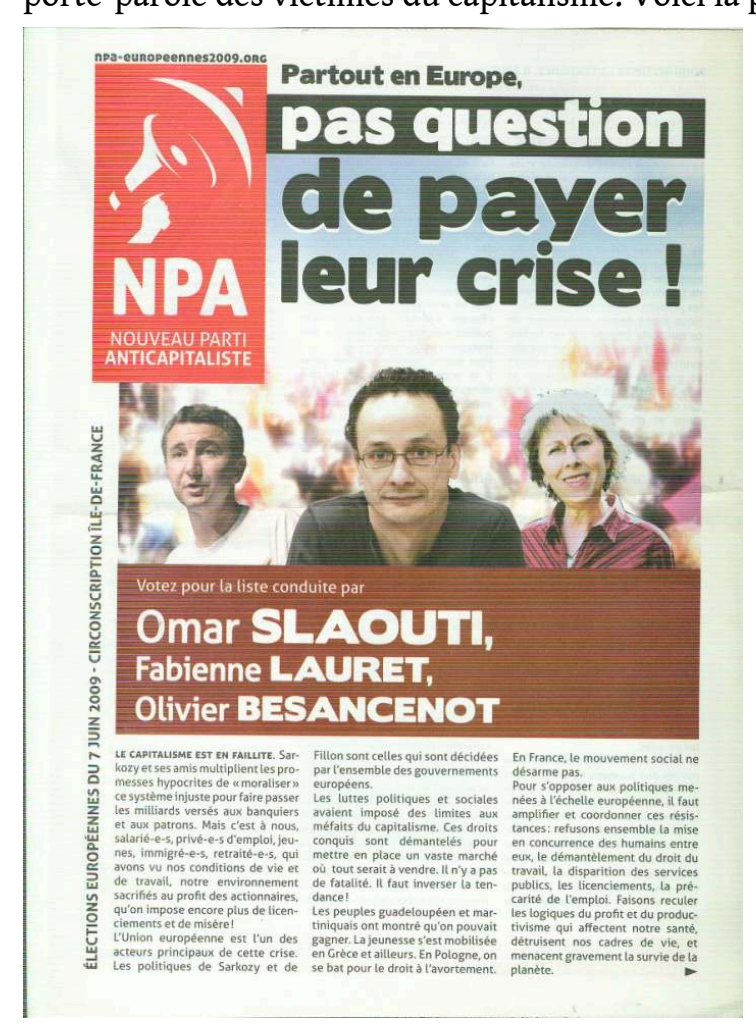

Le début du texte souligne une volonté de parler pour un ensemble limité d'électeurs : «C'est à nous, salarié-e-s, privé-e-s d'emploi, jeunes, immigré-e-s, retraité-e-s, qui avons vu nos conditions de vie et de travail, notre environnement sacrifiés au profit des actionnaires, qu'on impose encore plus de licenciements et de misère. "

Le «nous » marque l'appartenance des candidats au monde des dominés. Comme celui des candidats du Parti communiste de la Réunion, leur habillement incite le lecteur à construire un ethos de travailleur, au contact du monde de la « misère ", opposé à celui des «actionnaires ». Un détail qui a son importance : le nom d'Omar Slaouti se détache en lettres plus grandes et celui d'Olivier Besancenot, en lettres plus petites, est placé en dernière position. Or Besancenot est une figure reconnue du monde politique et de l'ensemble des électeurs', alors qu'Omar Slaouti est un parfait inconnu. Il s'agit de montrer, dans la perspective d'extrême gauche, que c'est le parti des travailleurs qui est l'acteur légitime, et non tel ou tel individu. D'autant plus que le nom d'Omar Slaouti 
semble montrer qu'il s'agit d'un travailleur immigré, avec tout ce que cela implique de positif pour les positionnements politiques de gauche.

Le logo du NPA contient le pictogramme d'un mégaphone dont la signification est double : faire du parti un simple amplificateur de la parole des travailleurs, et l'associer par métonymie aux cortèges de manifestants, de façon à mettre en évidence le rôle d'animateur de "luttes» sociales qu'entend jouer le parti. Mais pour être de bons médiateurs, les candidats du NPA doivent aussi, à travers leur profession de foi, tenir un discours qui les met à distance du monde de la "misère ", ils doivent se montrer membres d'un parti capable d'analyses politiques. On retrouve là l'ethos du militant marxiste qui par son savoir domine le fonctionnement du monde où il est dominé. Le porte-parole est ainsi soumis à une triple pression: il doit montrer une relative conformité à la parole des sans-voix, mais aussi une conformité au positionnement de son organisation et aux canons d'une parole politique légitime. Dans le texte on voit ainsi s'entrelacer trois registres distincts: 1) des traits d'oralité censés typiques de l'expression populaire : ainsi le slogan même qui domine tout le texte: «Pas question de payer leur crise "; 2) le respect des normes imposées par un certain genre du discours politique ; 3 ) des caractéristiques de la parole des mouvements d'extrêmegauche, perceptible à tous les niveaux du texte ( $\mathrm{du}$ vocabulaire au mode de raisonnement et au didactisme, en passant par une typographie relativement austère), comme en témoigne la seconde page du texte.

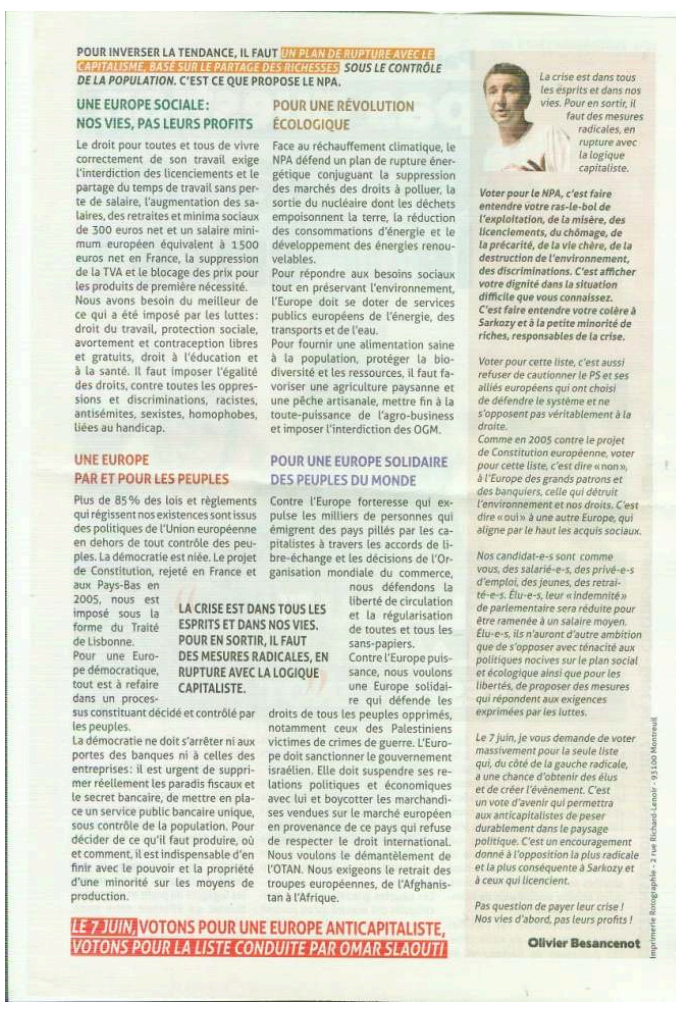

Si maintenant on se tourne vers la profession de foi du MODEM, le contraste est saisissant, en particulier en ce qui concerne les vêtements, qui marquent une appartenance au monde des professionnels de la politique, censés être au service de l'intérêt général et non de tel ou tel secteur de la population. 

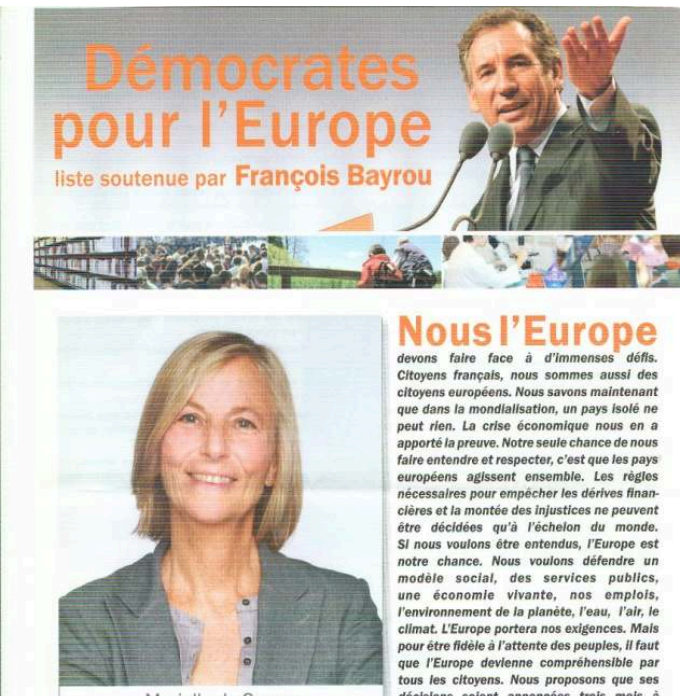

\section{Nousl'Europe} devons faire face à d'immenses déffs.
chtoyens françals, nous sommes aussi des citoyens européens. Nous savons mainteneant qeut rien. La crise économique nous en e apporté la preuve. Notre seule chance de nous taire ententre et respecter, c'est que les pays
européens a ajissent ensemble, Les rélilos

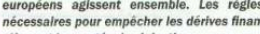
clères et ta montée des injustices ne peuvent

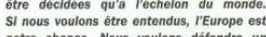
notre chance. Nous voulons défendre un
modele social, des services publics. $\begin{aligned} & \text { modèle social, des services publics, } \\ & \text { une économie vivante, nos emplots. }\end{aligned}$
a renvironnement de la planente, leau, l'air, le climat L'Europe portera nos exisences. Mais
pour être fidele à arttente des peuples, II faut

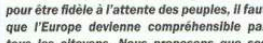
que r Europe devienne comprehensile ses
tous les cltotoven. Nous sprososons que ses
décisions soient annoncees trois mois

Marielle de Sarnez

Notre équipe de France pour l'Europe lledefirance : Marielle de SARNEZ, députee européer

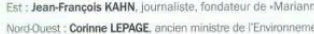

Su-Ouest : Robert ROCHEFORT, directeur du CREDOC

SudEst: Jean-Lue BENNAHMias. depputé européen

Duest: Syvie GOULARD,

Massit Centrat icentre : Jean-Marie BEAUPUY, obputé europeer l'avance pour que chacun puisse exprimer son avis par lintermédiaire des élus, des que toute délibiration devienne publique pour qu'on ne puisse plus dile - c'est la faute a Buxellies, Nous vous proposons une équipe
de candidats remarquables par leur

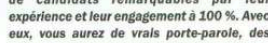
eux, vous aurez de vrals porte-parole, des
députés européens ecoutés et respectés. $\begin{aligned} & \text { Pour faire entendre, au } \\ & \text { Parlement europien, } \\ & \text { notre voix de citoyens. }\end{aligned}$
f. Bywm .

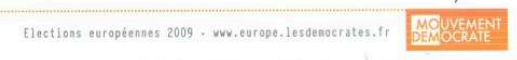

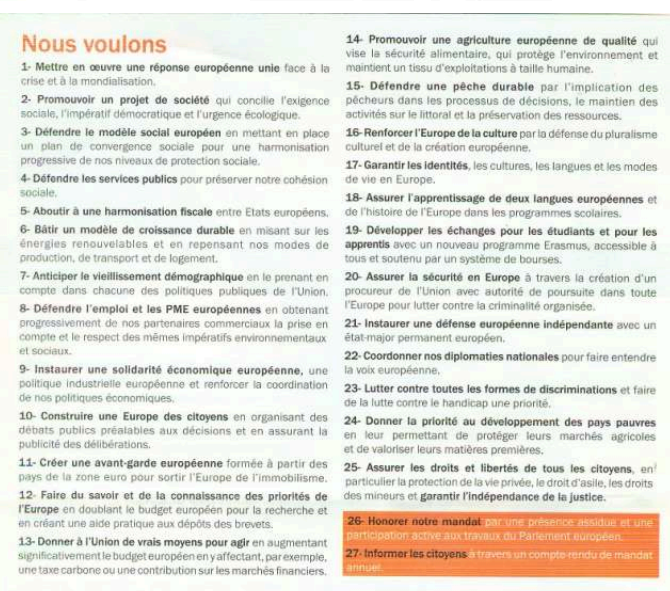
Autour de François Bayrou en Ile-de-France

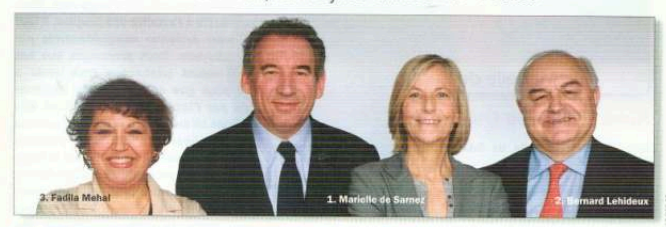

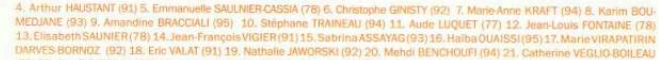

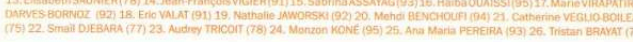
Electlons europeennes 2009 - www.europe, lesdemocrates.fr DEMOVEMEN

La conclusion du texte de la première page fait apparaître le terme "porte-parole »: «Nous vous proposons une équipe de candidats remarquables par leur expérience et leur engagement à $100 \%$. Avec eux vous aurez de vrais porte-paroles, des députés européens écoutés et respecté, pour faire entendre, au parlement européen, notre voix de citoyens. » 
Significativement, à la différence du NPA, le texte ne spécifie pas de quelle collectivité retreinte le MODEM est le «porte-parole». Il s'agit seulement du «vous»des " citoyens ". Même au NPA, le porte-parole a beau essayer de se montrer "inspiré ", travailleur comme les autres, porte-voix des victimes du capitalisme, il ne peut échapper aux contraintes du champ politique. Certes, c'est le nom d'Omar Slaouti qui ressort au milieu d'un collectif, mais le NPA n'entend pas se tenir dans le registre de la pitié : il inscrit les souffrants dans un système explicatif plus vaste qui en fait un acteur essentiel de la transformation sociale. Dans les énonciations politiques qui se présentent comme au service de sans-voix il se mêle ainsi inévitablement, dans des proportions variables, des caractéristiques de porte-parole "délégué » et de porteparole «inspiré ». Il faut sortir du champ proprement politique, évoquer des personnalités issues de la société civile pour que s'impose la figure du porte-parole « inspiré », du pur « porte-voix ».

\section{Le porte-voix}

Dans l'espace public contemporain, l'appel à la pitié mobilise régulièrement des portevoix issus du sport, de la chanson, de la mode, du cinéma, de la littérature..., qui mettent leur notoriété au service de sans-voix. On ne leur demande pas d'appartenir au monde de ceux qu'ils défendent mais d'apparaitre dans les médias pour montrer leur volonté d'engagement, leur sens des responsabilités, leur humanité en exprimant leur émotion devant de telles souffrances.

\subsection{Ambassadrice de bonne volonté}

On peut évoquer l'exemple de l'actrice Angelina Jolie, qui s'était vu conférer en 2001 par le Haut-Commissariat des Nations Unies pour les réfugiés, le titre de «Goodwill Ambassador » pour les réfugiés.

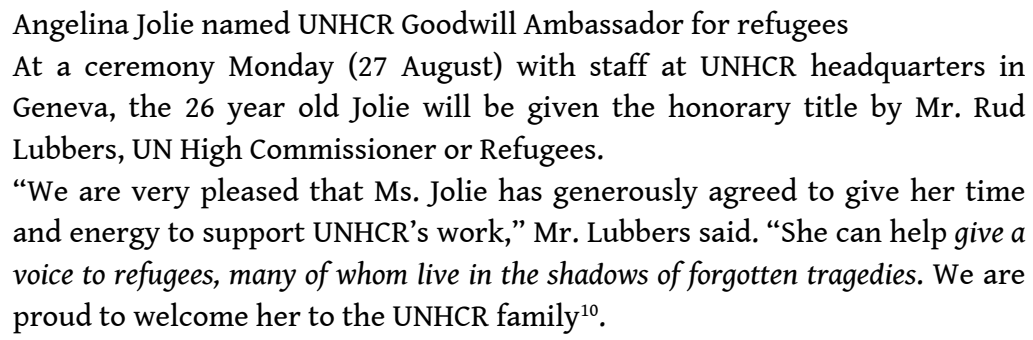

Le nom «ambassadrice» oriente le porte-voix vers le statut de porte-parole officiel rattaché à la sphère haute, tandis que le complément « de bonne volonté » oriente vers le charisme, l'inspiration. "Good will» figure d'ailleurs dans l'Evangile de la Nativité, quand les anges chantent «Glory to God in the highest, and on earth peace, good will toward men. » (Luc, 2, 14) et en français « Paix aux hommes de bonne volonté ». ${ }^{11}$ Lors de la cérémonie d'investiture, c'est précisément le rôle de porte-voix des sans-voix qui a été mis en avant par le Haut-Commissaire: A. Jolie doit "donner une voix " (give a voice) à des personnes qui sont invisibles, oubliées (« in the shadows of forgotten tragedies »). En réalité, il s'agit non de donner une voix, mais de convertir le murmure indistinct des souffrants en parole qui peut accéder à la sphère haute, énoncée en 
anglais, dans la langue des dominants, par une locutrice qui est maximalement visible et audible.

Certes, ces porte-voix médiatiques ne sont pas censés appartenir au monde de ceux qu'ils défendent, mais pour autant ils ne peuvent pas laisser se creuser un trop grand écart avec eux. Ici encore l'habillement joue un rôle important dans un univers médiatique où ce sont avant tout des images qui circulent. Dans les camps de réfugiés, Angélina Jolie s'habille très simplement et essaie d'adopter certains traits vestimentaires de ceux à qui elle doit " donner une voix ». La riche iconographie de ses visites aux camps de réfugiés est à cet égard révélatrice. C'est précisément l'écart entre les toilettes " glamour ", caractéristiques de scènes emblématiques telles que la montée des marches au Festival de Cannes, et l'ethos vestimentaire de simplicité qui souligne l'engagement du porte-voix : il partage l'humanité des souffrants.

\subsection{De la chanson du sans-foyer au rock des sans-voix}

Nous aimerions à présent envisager un cas d'appel à la pitié en faveur des sans-voix de nature différente, dans la mesure où il mobilise des ressources esthétiques. Ce qui ne va pas sans soulever des difficultés: comment peut-on produire des textes ayant une valeur artistique sans sortir du registre de l'appel à la pitié ?

Il existe un nombre considérable d'œuvres littéraires où l'auteur cherche à attirer la sympathie du lecteur sur des individus présentés comme des «sans - ». Il suffit de songer au monument que représentent Les misérables de Victor Hugo ou Germinal de Zola. La chanson n'est pas en reste ; ses capacités de diffusion sont d'ailleurs beaucoup plus grandes celles des œuvres proprement littéraires. Au début du $20^{\circ}$ siècle, par exemple, une chanson patriotique à succès, "L'homme aux guenilles »" ${ }^{12}$, mettait en scène "un sans-foyer, sans-famille ». Les deux premières sections de la chanson le décrivent à la non-personne :

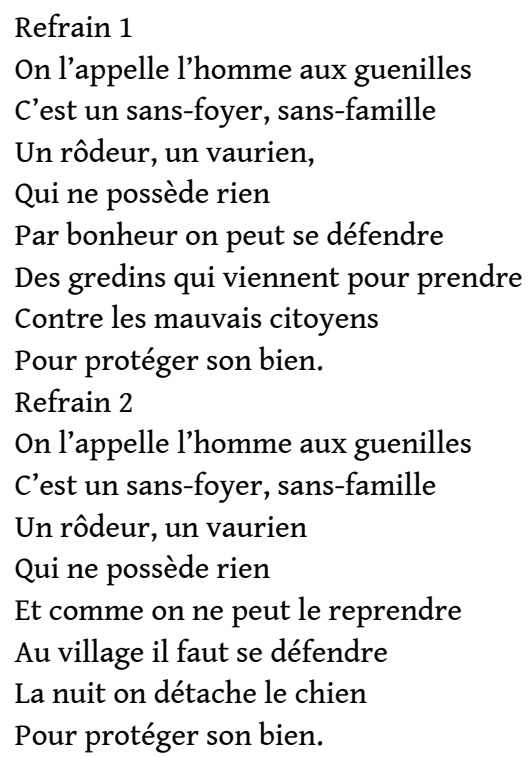

37 Arrêté par des soldats prussiens qui menacent de le tuer s'il ne donne pas des renseignements, il s'écrie fièrement : 


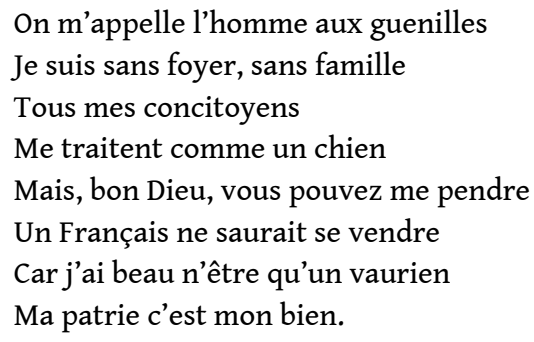

\section{Fondation Abbé Pierre. Son dossier de presse explique le projet qui l'anime :}

Ce groupe musical varois né en février 2014 est le fruit de la rencontre entre Piero SAPU, figure de la scène alternative (chanteur des Garçons Bouchers, BB Doc, Docteur Destroy), et tous les «Sans », les « oubliés » de notre société, dont le groupe SANS VOIX s'est donné pour objectif d'amplifier et porter la parole.

L'originalité du projet ? Une voix du rock francophone décide de mettre son talent de parolier et son charisme scénique (plus de 15 ans de scène !) au service des délaissés, devenant ainsi amplificateur et porte-voix.

Les paroles des morceaux composés par le groupe SANS VOIX (une vingtaine de compositions à ce jour) sont les mots des "galériens " de l'existence, recueillis lors d'ateliers d'écriture, dans des livres, ou au gré de rencontres à travers la France.

Rachel, Henri, Catherine, William..., sont les Sans-Voix croisés dans les associations humanitaires du Var ou d'ailleurs, dont Piero SAPU clame fièrement la parole!

(http://www.sansvoix.fr/presse/ : «Dossier de présentation à télécharger »; consulté le 9/2/2018)

Piero Sapu n'est pas un porte-voix médiatique qui - à l'instar d'Angelina Jolie - serait étranger au monde des sans-voix. Comme les autres membres du groupe, il est censé vivre au plus près de ceux qu'il défend. Néanmoins, le texte révèle une tension. « Les 
Sans Voix » sont présentés à la fois comme des " porte-parole des exclus » et comme la « rencontre » entre un chanteur connu et les "Sans », les « oubliés de notre société ». Le collectif apparaît ainsi centré sur un chanteur qui « décide de mettre son talent de parolier et son charisme scénique (plus de 15 ans de scène !) au service des délaissés ». Un tel choix va aux devant des attentes des médias, qui ont besoin d'incarner les collectifs dans l'image d'un individu. L'apparence du chanteur, Piero Sapu - chauve, tatoué et barbu - correspond au stéréotype de l'artiste marginal vivant parmi les pauvres. Comme on peut s'y attendre, c'est lui, et non l'ensemble du groupe, qui a été au cœur d'un reportage consacré aux "Sans Voix » dans une émission de télévision « Toute une histoire », diffusée par la chaîne nationale France 2, le 21 mars 2015.

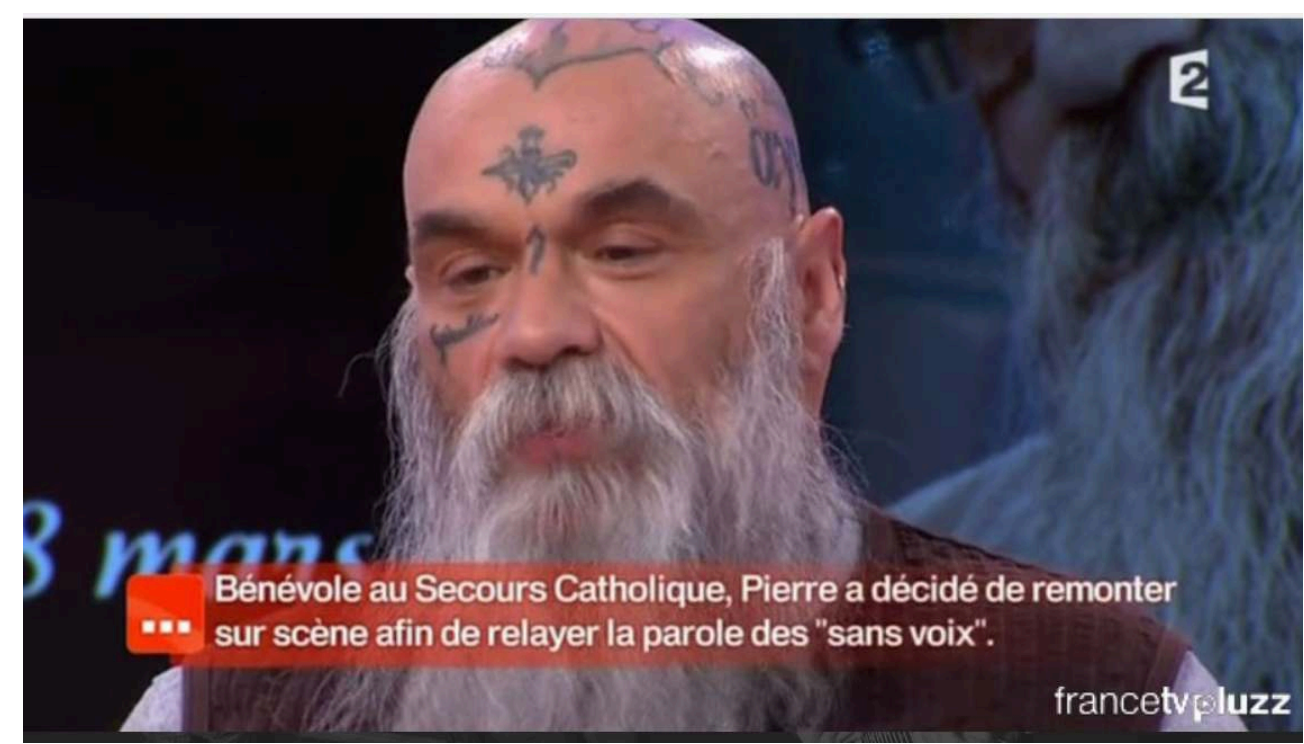

(https://www.sansvoix.fr/presse/ ; consulté le 22/6/2018)

Pour ne pas trop s'éloigner des « sans voix », et donc ne pas se délégitimer, le groupe ne peut pas véritablement s'ancrer dans le champ musical : des textes ou une musique qui seraient très élaborés sur le plan esthétique seraient difficilement compatibles avec l' ethos d'individus qui veulent "relayer la parole des sans voix ». Figure d'ailleurs en bonne place sur le site Web du groupe ${ }^{13}$ le mégaphone, qui, rappelons-le, constitue aussi le logo du Nouveau Parti Anticapitaliste : le groupe entend montrer qu'il ne fait qu'amplifier une parole déjà là, inaudible dans la sphère haute. Cette préoccupation affleure dans le dossier de presse. Les textes des chansons sont en effet présentés comme produits collectivement («Les paroles des morceaux composés par le groupe SANS VOIX »), et " recueillis lors d'ateliers d'écriture, dans des livres, ou au gré de rencontres à travers la France ». Ils seraient donc une transposition des paroles des sans-voix concernés.

Les paroles des chansons doivent ainsi sembler élémentaires, montrer l'ethos des laissés pour compte. La première strophe d'une des chansons phares du groupe, " Rachel », est significative à cet égard. Elle montre un ethos simple, à la mesure de la personne simple qu'elle évoque, tout en thématisant la coupure entre sphère haute et sphère basse, qui justifie l'existence même du groupe.

Ce n'est qu'une voix qu'on ne voit pas qu'on ne regarde pas

Une petite voix une simple voix qui n'existe presque pas

Ce n'est qu'une voix qu'on n'entend pas qu'on n'écoute plus ou pas 
Il en va de même pour la chanson « Underground » :

T'as pas vraiment le choix quand t'es underground Du comment, du pourquoi quand t'es underground Pas la tête de l'emploi quand t'es underground T'as pas vraiment le choix... ${ }^{14}$

Le dossier de presse a beau présenter les textes de ces chansons comme des mots "recueillis lors des ateliers d'écriture », on repère aisément le mouvement réflexif par lequel les paroles légitiment leur propre énonciation : la chanson « Rachel » ne fait que gloser le sens de "sans-voix », le nom même du groupe. De son côté, "Underground " développe la même thématique sur une autre isotopie : le sans-voix est invisible parce qu'il est under-ground, sous terre, il appartient à la sphère basse, et cette énonciation même a pour visée de le rendre visible.

Si l'élaboration esthétique était poussée plus avant et si les membres du groupe agissaient comme de vrais professionnels, on basculerait dans le registre de la chanson de variété. C'est d'ailleurs ce qui se passe pour ceux qui se présentent comme appartenant à un monde d'exclus, mais exercent leur activité à l'intérieur du champ proprement musical. On pense en particulier au rap.

\subsection{La chanson des Enfoirés}

Nous allons évoquer une autre manière de résoudre cette tension entre esthétique et appel à la pitié : la chanson des «Enfoirés ». Le 26 septembre 1985, l'acteur comique Coluche lance sur la radio privée Europe 1 l'idée des « Restos du Cœur »:
J'ai une petite idée comme ça. Si des fois y a des marques qui m'entendent, y a des gens qui sont intéressés pour sponsoriser une cantine gratuite qu'on pourrait commencer par faire à Paris... nous on est prêts à aider une entreprise comme ça qui ferait un resto qui aurait comme ambition, au départ, de distribuer deux ou trois mille couverts par jour...

Coluche cumule ici le rôle d'Angelina Jolie et de celui d'un fondateur d'organisation caritative. Il demande à un autre artiste à succès, le chanteur Jean-Jacques Goldman, de faire une chanson pour diffuser le message. Ce dernier compose La Chanson des Restos, qui sort en disque 45 tours ; 533900 exemplaires sont immédiatement vendus au profit de l'association.

50 Ce qui nous intéresse ici est de voir comment cette chanson, bien qu'écrite par une star, s'efforce de ne pas basculer dans l'univers esthétique. Une chose qui contribue à la " désesthétiser » est le caractère collectif de l'interprétation; une autre est le fait que dans ce groupe ne figurent que deux chanteurs, ou plutôt un chanteur et demi : les «Enfoirés» rassemblent des acteurs (Coluche, Nathalie Baye), un chanteur (JeanJacques Goldman), un acteur-chanteur (Yves Montand), un footballeur (Michel Platini) et un animateur de télévision (Michel Drucker). Le fait que la plupart ne soient pas des chanteurs vient assoir le présupposé pragmatique que dans ce texte la visée esthétique n'est pas première, que c'est l'urgence qui contraint à intervenir ceux qui n'ont pas vocation à le faire. Troisième facteur de "désesthétisation»: le texte n'est pas intégralement chanté. Seul le refrain l'est, et par l'ensemble des participants.

51 Voici le premier couplet, qui est suivi du refrain : 


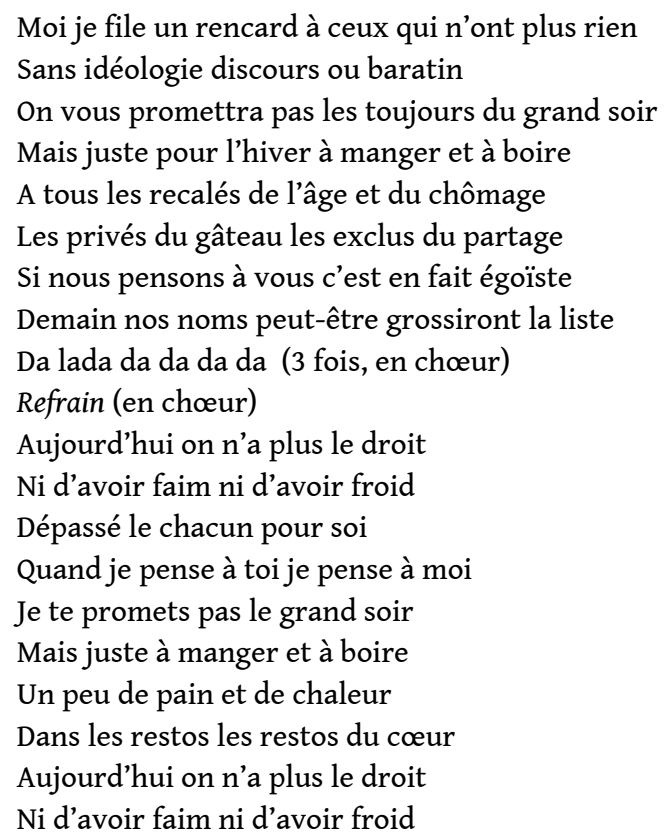

52 Les quatre premiers vers sont dits par Coluche, les quatre suivants par Yves Montand: personne ne doit s'approprier le texte. Les « enfoirés » ont beau être des célébrités, ils participent à une entreprise qui se place en quelque sorte entre parenthèses de leurs carrières respectives, individuelles. Le fait que les couplets soient dits et non pas chantés se charge de valeur morale dans le cadre d'un appel à la pitié : la simple voix est à la mesure de la souffrance. Chacun parle avec la voix qu'on lui connaît, déjà bien identifiée dans les médias, avec son humanité, qu'il partage avec les souffrants. Les vrais chanteurs (Goldmann et Montand) ne sont pas distingués des autres. Tout est conçu pour éviter une mise en spectacle valorisante de l'interprète. Le chant est réservé au refrain, qui relègue à l'arrière-plan sa dimension esthétique par son caractère collectif, incarnation de la solidarité, et par son contenu, réduit à un « Da lada da da da da », pure expression d'un affect.

53 Pas plus que «Les Sans Voix", les «Enfoirés» ne peuvent produire des textes sophistiqués, assumer des énoncés qui afficheraient des marques de littérarité trop fortes. Un investissement esthétique trop prononcé risquerait à tout moment d'être perçu comme un artifice mensonger, incompatible avec la vérité du cœur, l'authenticité d'une âme compatissante. C'est toute la différence avec un texte comme «L'homme aux guenilles", où les auteurs entendent faire œuvre, en déployant un univers fictif et en mettant en évidence de multiples signes de poéticité. La chanson des Restos du cœur ne raconte pas une histoire, mais sollicite directement la générosité en mobilisant un français standard, voire familier, tant pour le lexique que pour la syntaxe. On peut toujours objecter qu'en réalité le texte a été écrit par un professionnel, que la simplicité est une stratégie rhétorique comme une autre, mais il faut bien prendre acte de la prétention énonciative de la chanson.

\section{Une vidéo}

Entre le cas de figure que représentent le rock des «Sans Voix » et les « Enfoirés », qui s'efforcent de se «désesthétiser» pour se maintenir dans le registre de l'appel à la 
pitié, et les textes qui, comme "L'homme aux guenilles ", s'inscrivent pleinement dans le champ esthétique il existe des situations intermédiaires. C'est le cas par exemple d'une vidéo ${ }^{15}$ intitulée "Stop au déni. Les sans voix. 19 témoignages anonymes de victimes de violences sexuelles, lus par 19 personnalités ».

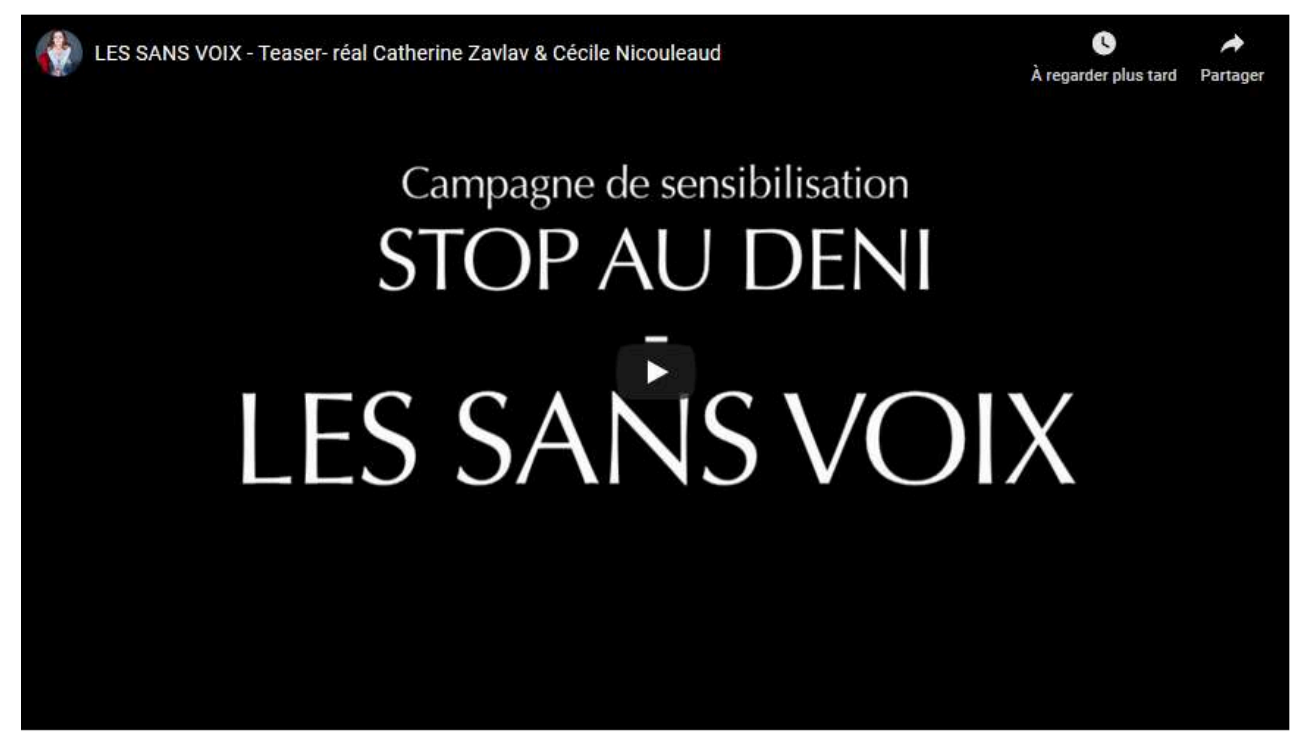

Cette vidéo est en réalité le "teaser ", largement diffusé en 2015, d'un film dont le projet est ainsi décrit sur le site du magazine féminin Marie-Claire :

Pparce que " les victimes deviennent trop souvent des sans voix ", des personnalités prennent la parole en lisant des témoignages, pour donner la parole à celles et ceux ayant subi des violences sexuelles.

Ce teaser du film "Les Sans Voix », réalisé par Catherine Zavlav et Cécile Nicouleaud dans le cadre de la campagne «Stop au déni » dévoile une série de témoignages de victimes d'abus sexuels, lus par des personnalités. Un face-à-face avec la caméra qui interpelle le spectateur, le met devant une réalité qui effraie et dérange.

Le film « Les Sans Voix », sera diffusé en avant-première le 2 mars 2015, lors du colloque «Enquête de reconnaissance» organisé par l'association Mémoire Traumatique et Victimologie au Palais du Luxembourg. Il fait la lumière sur l'impact de ce type de violences de l'enfance à l'âge adulte. Quel avenir et quel traitement pour les victimes ? Comment vivre avec cette douleur, « cette bombe à retardement » qui ronge de l'intérieur.

Il faut entendre les victimes pour briser les tabous, déclarer «la guerre au déni de violences sexuelles».

"Un changement d'état d'esprit de la société face à ses violences intimes » est nécessaire pour reconnaître aux personnes ayant subi des violences le statut de victime.

(https://www.marieclaire.fr/,stop-au-deni-la-video-qui-donne-la-paroleaux-victimes-de-violences-sexuelles,733908.a ; consulté le 10/1/2019)

Il n'y a pas véritablement ici d'appel à la pitié qui inciterait les destinataires à faire quelque chose de précis en faveur des sans voix, comme c'est le cas avec la chanson des Restos du cœur. Le but est plutôt d'attirer la sympathie sur une certaine catégorie de souffrants, d'influer sur l'opinion, de contribuer « à un changement d'état d'esprit de la société face à ses violences intimes ». des personnalités qui ne sont pas toutes connues d'un public large. Ils disent face à la 
caméra des « témoignages » censés écrits par des « sans voix », victimes d'abus sexuels. Ils ne sont même pas nommés; ce sont littéralement des porte-voix, qu'on ne peut en aucun cas identifier avec l'auteur de ce qu'ils lisent : un homme peut dire le témoignage d'une femme, et réciproquement. Ils se succèdent, sans même qu'on puisse savoir s'il s'agit du même témoignage ou de témoignages distincts. L'idée est de créer une sorte de voix anonyme et indifférenciée. Voici le début :

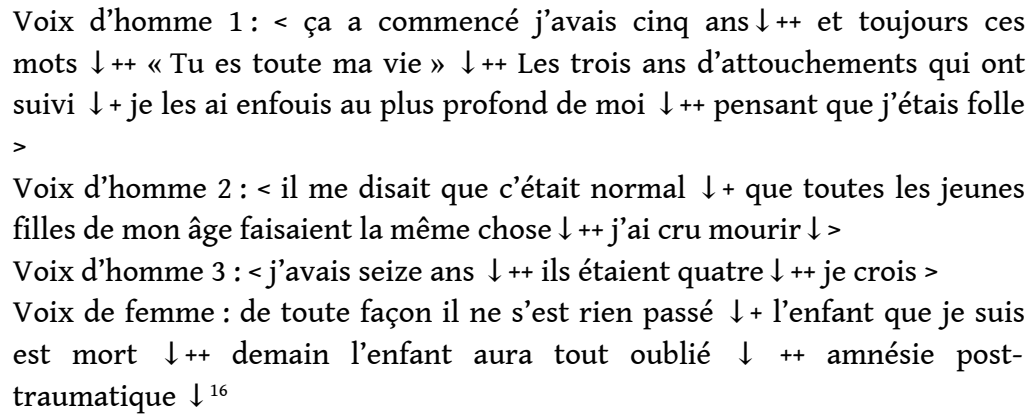

Les interprètes ont manifestement reçu pour consigne d'adopter un ethos distancié, une voix monocorde qui résulte du recours systématique à un débit lent, une intonation descendante et de nombreuses pauses. La scénographie, très sobre, ne montre que leur tête, qui se détache sur un fond noir. Ce dispositif polyphonique permet

- un effet d'authenticité : le locuteur ne doit chercher ni se singulariser, ni à séduire, bref à se donner en spectacle ;

- une homogénéisation: les "sans voix" apparaissent comme étant en un sens la même victime, par-delà la diversité des témoignages ;

- un contraste entre le contenu et l'énonciation: le pathétique de ce qui est dit est rendu plus fort par le fait que l'énonciateur adopte un ethos distancié.

On pourrait être tenté de parler ici d'un effacement d'ethos (Maingueneau 2014), mais cet effacement n'est qu'apparent; il vise à provoquer une "incorporation " (Maingueneau 1983, 1999) paradoxale : les victimes sont " mortes », comme d'ailleurs le donne à entendre l'image où s'affiche le titre de la vidéo : un rectangle noir et des lettres blanches. Seule une voix d'outre-tombe est censée être à la mesure de leur souffrance; comme ces «sans voix » ont perdu la parole, il faut que d'autres portent leur voix, en s'effaçant, en s'attachant à faire entendre l'indicible.

60 La vidéo fait interagir trois niveaux d'énonciation : 1) les témoignages des souffrants, 2) la parole des locuteurs qui les disent, 3) l'œuvre des deux auteures qui la signent. On peut effectivement parler d'œuvre. Il n'est pas besoin d'avoir une grande familiarité avec la littérature contemporaine pour percevoir à quel point le matériau de départ a été réélaboré. On a l'impression de se trouver dans un univers vocal proche de celui des films de Marguerite Duras ${ }^{17}$. Il y a manifestement tension entre l'intention revendiquée de donner la parole aux sans-voix et cette forte esthétisation. Cela tient sans doute pour une part au fait que ces "sans voix » ne sont pas des exclus de la sphère haute, mais des exclus de la parole : s'ils sont sans voix, c'est parce qu'ils ont honte de parler ou sont trop traumatisés pour le faire. La mobilisation de ressources esthétiques s'impose plus naturellement quand on se confronte à l'indicible plutôt qu'à l'inaudible de paroles venues de la sphère basse. 


\section{Conclusion}

61 J'ai restreint mon propos à des textes qui circulent dans l'espace public et dont la visée principale est d'appeler à la pitié. Dans ce vaste ensemble je me suis intéressé à un certain type de souffrants, les « sans-voix », qui appellent l'intervention de médiateurs, de « porte-voix ». Au lieu de délimiter un dispositif d'énonciation stable, on a affaire à une zone floue où peuvent s'élaborer des dispositifs très divers, dont les frontières sont d'une part le discours politique, d'autre part le champ esthétique.

62 Ces deux frontières ne sont pas de même nature. On voit mal comment un texte d'appel à la pitié pourrait ne pas mobiliser de ressources esthétiques, à partir du moment où il doit agir sur la sensibilité d'un vaste public et activer des valeurs. Le problème pour les locuteurs est alors de neutraliser par divers moyens les aspects de la communication esthétique difficilement compatibles avec le type d'appel à la pitié qu'ils entendent activer. Quant au discours politique, rien n'empêche qu'il fasse massivement appel aux émotions et ne se tourne pas vers des "sans-voix "; mais dans ce cas la pitié va au-delà de la pitié : elle suppose une explication fondée sur une analyse de la société dont la validité est garantie par un appareil et une doctrine. On ne met pas directement les destinataires au contact des souffrants à travers une émotion qui atteste de l'appartenance à une même humanité.

\section{BIBLIOGRAPHY}

Aubin, France. 2009. « La figure de l'intellectuel porte-parole en milieu culturel collectiviste », Quelle communication pour quel changement? Les dessous du changement social, Christian Agbobli (éd.) (Québec : Presses de l'Université du Québec), 252-260 (disponible à l'adresse http://www.puq.ca/ catalogue/livres/quelle-communication-pour-quel-changement-1735.html)

Brodiez-Dolino, Axelle, Isabelle von Bueltzingsloewen, Benoît Eyraud, Christian Laval \& Bertrand Ravon (éds). 2014. Vulnérabilités sanitaires et sociales. De l'histoire à la sociologie (Rennes : Presses Universitaires de Rennes)

Butler, Judith.2004 [1997]. Le pouvoir des mots. Politique du performatif, trad. fr. (Paris : Editions Amsterdam)

Charaudeau, Patrick. 2000. "La pathémisation à la télévision comme stratégie d'authenticité », dans Les émotions dans les interactions (Lyon : Presses Universitaires de Lyon)

Charaudeau, Patrick. 2001. La télévision et la guerre. Déformation ou construction de la réalité ? Le conflit en Bosnie (1990-1994) (Bruxelles : Ina-De Boeck)

Gautier, Claude. 2015. « La voix des sans-voix. Condamnés à être parlés ? La condition du porteparole ", Langages, politique, histoire. Avec Jean-Claude Zancarini, Descendre, Romain \& Jean-Louis Fournel (éds) (Lyon : ENS éditions), 587-598

Guilhaumou, Jacques. 1998. La parole des Sans. Les mouvements actuels à l'épreuve de la Révolution française (Paris : ENS Éditions) 
La Mantia, Francesco. 2016. « La strategia del ventriloquo. Sul portavoce », Revista di Filosofia del Lenguaggio 10: 2, 153-164

Maillard, Nathalie.2011. La vulnérabilité, une nouvelle catégorie morale ? (Genève : Labor \& Fides)

Maingueneau, Dominique.1998. Analyser les textes de communication (Paris : Armand Colin)

Maingueneau, Dominique.1999. « Ethos, scénographie, incorporation », Images de soi dans le discours, La construction de l'ethos, Ruth Amossy (éd.), Lausanne, Delachaux \& Niestlé, 75-101

Maingueneau, Dominique. 2004. « Hyperénonciateur et 'particitation' », Langages 156, 111-127

Maingueneau, Dominique. 2012. Les phrases sans texte (Paris : Colin)

Marcoccia, Michel. 2004. « Le paradoxe du porte-parole », in Delamotte-Legrand, Régine (éd.), Médiations langagières, vol. 2 (Rouen : Publication de l'Université de Rouen), 146-166

Paveau, Marie-Anne. (2017). « Le discours des locuteurs vulnérables. Proposition théorique et politique », Cadernos de Linguagem e Sociedade $18: 1,135-157$

Payet, Jean-Paul. 2011. «L'enquête sociologique et les acteurs faibles », SociologieS [En ligne], http://journals.openedition.org/sociologies/3629

Payet, Jean-Paul, Giuliani, F. \& Laforgue, D. (éds). 2008. La voix des acteurs faibles. De l'indignité à la reconnaissance (Rennes : Presses Universitaires de Rennes)

Rabatel, Alain, 2016, « Analyse de discours et inégalités sociales : de l'empathie pour les invisibles à l'engagement pour le commun », Revista de Estudos da Linguagem 24 :3, 757-788

Spivak, Gayatri Chakravorty. 2006 [1988]. Les subalternes peuvent-elles parler?, trad. fr. (Paris :

Editions Amsterdam)

Thomas, Hélène. 2010. La vulnérabilité. La démocratie contre les pauvres (Paris : Éditions du Croquant)

Tronto, Joan. 2009 [1993]), Un monde vulnérable. Pour une politique du care (Paris : La Découverte)

\section{NOTES}

1. 21-22 juin 2018 ; colloque organisé par D. Ducard, B. Ferron, E. Née, C. Oger

2. Sur cette question on peut se reporter à l'article de'E. Soares.

3. C'est nous qui soulignons.

4. C'est nous qui soulignons.

5. Voir par exemple Charaudeau (2000, 2001).

6. Sur le porte-parole, voir en particulier Marcoccia (2004), Aubin (2009), Gautier (2015), La Mantia (2016).

7. Sur le rôle du préfixe "sans" dans un certain nombre de mouvements sociaux, voir par exemple Guilhaumou (1998), qui appuie sa réflexion sur le modèle fourni par les «sans-culottes » de la Révolution française.

8. Il s'agit d'un texte de quatre pages qui est envoyé par les pouvoirs publics à tous les électeurs quelques jours avant l'élection. Les professions de foi des divers candidats sont regroupées dans une même enveloppe.

9. Il a recueilli 4,08\% des suffrages aux élections présidentielles de 2007.

10. C'est nous qui soulignons. http://www.unhcr.org/news/press/2001/8/3b85044b10/angelinajolie-named-unhcr-goodwill-ambassador-refugees.html ; communiqué de presse du 23 août 2001. Consulté le 15 décembre 2018. 
11. C'est nous qui soulignons. Je cite ici les traductions traditionnelles, pour l'anglais celle de la Bible du roi Jacques et pour le français celle de la Vulgate ( in terra pax hominibus bonae voluntatis »). Ce sont elles qui sont passées dans l'usage. On notera que l'Eglise catholique préfère aujourd'hui traduire par " paix aux hommes qu'il aime».

12. Paris : Aux succès modernes, 1915 ; paroles de Léon Joreb, musique de Henri Piccolini

13. https://www.sansvoix.fr/album/ ; consulté le 17 février 2017

14. https://www.sansvoix.fr/album/ ; consulté le 10 février 2019. On notera que les chansons sont présentées comme une élaboration collective. Pour « Rachel » le site donne la liste suivante : «Rachel Boncoeur / W. Delgado, L. Gasnier, G. Mas, L. Merle, C. Parel»; pour « Underground : « Dominique Remond / L. Gasnier, G. Mas, L. Merle, C. Parel ». Il s'agit d'affaiblir autant que faire se peut la souveraineté d'une position d'auteur.

15. Accessible sur Youtube : https://www.youtube.com/channel/UCJ9rpGASyzJQU8U4p5GpiMg

16. Conventions de transcription: $\downarrow$ intonation descendante, + pause brève, ++ pause moyenne, $<$...> séquence prononcée avec un débit lent.

17. On notera que l'un des porte-voix de la vidéo, l'acteur M. Lonsdale, est un des personnages principaux d'India song, film culte de M. Duras.

\section{ABSTRACTS}

In this article we analyze a specific kind of appeal to pity in the media: the appeal to pity in favor of people who are presented as "voiceless" and, accordingly, need mediators. First, we emphasize the difference between this kind of mediation and political interventions in favor of groups that are considered outcasts. Then, we analyze the problems that arise when those who appeal to pity mobilize aesthetic resources. This point is illuminated by various contemporary examples: a charitable rock band whose name is "The voiceless", the first song in favor of "Les restos du Coeur" (a kind of French "soup kitchen" organization), and a video staging speakers who report testimonies gathered from interviews of "voiceless" persons that were sexually abused.

Dans cet article, nous cherchons à étudier un certain type d'appel à la pitié dans les médias en faveur de personnes qui sont présentées comme des "sans-voix", et qui, en conséquence, ont besoin de médiateurs. Dans un premier temps, nous soulignons la différence entre ce type de médiation et les interventions politiques en faveur de groupes considérés comme exclus. Puis nous analysons les difficultés qui surgissent quand ceux qui appellent à la pitié mobilisent des ressources d'ordre esthétique. Cette question est illustrée par l'étude de divers exemples empruntés au monde contemporain: un groupe de rock caritatif dont le nom est «Les Sans voix ", la Chanson des "Restos du cœur", et une vidéo où sont mis en scène des locuteurs qui sont censés rapporter des témoignages recueillis auprès de « sans-voix » victimes d'abus sexuels.

\section{INDEX}

Mots-clés: appel à la pitié, chanson, compassion, médiateur, porte-parole, sans-voix, sphère basse, sphère haute

Keywords: appeal to pity, compassion, high sphere, low sphere, mediator, spokesperson, song, voiceless 
AUTHOR

DOMINIQUE MAINGUENEAU

Sorbonne-Université 